\title{
Energy versus Information Based Estimations of Dissipation Using a Pair of Magnetic Colloidal Particles
}

\author{
S. Tusch, ${ }^{2}$ A. Kundu, ${ }^{1}$ G. Verley, ${ }^{1}$ T. Blondel,${ }^{1}$ V. Miralles, ${ }^{2}$ D. Démoulin, ${ }^{2}$ D. Lacoste,,${ }^{1}$ and J. Baudry ${ }^{2}$ \\ ${ }^{1}$ Laboratoire de Physico-Chimie Théorique, UMR CNRS Gulliver 7083, ESPCI, 10 rue Vauquelin, F-75231 Paris, France \\ ${ }^{2}$ Laboratoire LCMD, ESPCI, 10 rue Vauquelin, F-75231 Paris, France \\ (Received 12 December 2013; revised manuscript received 1 April 2014; published 9 May 2014)
}

\begin{abstract}
Using the framework of stochastic thermodynamics, we present an experimental study of a doublet of magnetic colloidal particles that is manipulated by a time-dependent magnetic field. Because of hydrodynamic interactions, each bead experiences a state-dependent friction, which we characterize using a hydrodynamic model. In this work, we compare two estimates of the dissipation in this system: the first one is energy based since it relies on the measured interaction potential, while the second one is information based since it uses only the information content of the trajectories. While the latter only offers a lower bound of the former, we find it to be simple to implement and of general applicability to more complex systems.
\end{abstract}

DOI: 10.1103/PhysRevLett.112.180604

In the last decade, a broad number of works have significantly improved our understanding of the thermodynamics of small systems. A central idea, namely the application of thermodynamics at the level of trajectories, has developed into a field of its own now called stochastic thermodynamics [1-4]. Manipulated colloids are a paradigmatic example of stochastic thermodynamics because of the ease with which colloids can be manipulated and observed.

Many studies of such systems have used a single colloidal particle, in a harmonic [5] or anharmonic potential $[3,6]$, which is described by an overdamped Langevin equation with a constant diffusion coefficient. Recently, Celani et al. have pointed out that the overdamped Langevin description fails to capture some aspects of the thermodynamics of this system in the presence of multiplicative noise due to temperature gradients [7]. In soft matter systems, temperature gradients are difficult to control at the micron scale, but multiplicative noise arises frequently due to hydrodynamic friction. In this Letter, we study such a case using a pair of magnetic colloids that are manipulated by a time-dependent magnetic field. This system offers a convenient mean to measure forces in various soft matter and biological systems because the colloids can be embedded in complex fluids or molecules of interest can be grafted on them [8].

In this Letter, we focus on a pair of bare manipulated colloids in water. In the first part, we evaluate the work distribution in this system within stochastic thermodynamics. In the second part, we evaluate an information theoretic bound for the dissipation in this process using only trajectory information.

The projection of the Brownian motion of both beads is observed in the plane parallel to the bottom wall with video microscopy. We assume that the fluctuations perpendicular
PACS numbers: 05.20.Jj, 05.40.Ca, 05.70.Ln, 82.70.Dd

to the wall are negligible since the beads have settled under gravity. Therefore, we focus on the two-dimensional relative displacement vector in polar coordinates $\mathbf{r}=(r, \theta)$, as shown in Fig. 1.

The interaction between the beads is modeled using a potential, which is the sum of three contributions: the dipolar interaction of the magnetic beads with each other $U_{\text {dip }}$, the interaction $U_{\text {mag }}$ of the beads with the applied
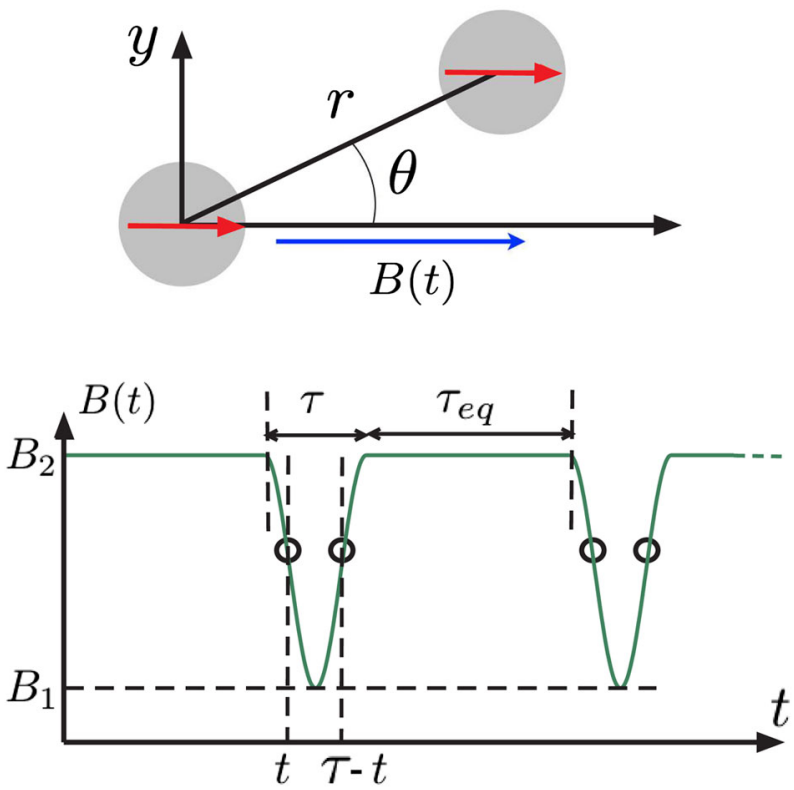

FIG. 1 (color online). Sketch of the experiment showing the two magnetic beads (top) and the time dependence of the applied magnetic field $B(t)$ (bottom). The protocol is composed of driving phases of duration $\tau$ followed by pauses of duration $\tau_{\text {eq }}$. The open circles represent two symmetric points at times $t$ and $\tau-t$ in this protocol. 
magnetic field $\mathbf{B}=B \hat{z}$, and a repulsive interaction of electrostatic origin $U_{\mathrm{el}}$ :

$$
U(r, \theta, B)=U_{\mathrm{dip}}(r, \theta, B)+U_{\mathrm{mag}}(B)+U_{\mathrm{el}}(r) .
$$

This potential has a short range repulsive part due to electrostatics and a long-range attractive part due to dipolar interactions as described in [9] and in the Supplemental Material [10]. First, we prepare the system in an equilibrium state in a constant magnetic field. In this case, the distribution of the relative coordinate should follow a Boltzmann distribution, which we use to test our model. We manage to obtain a very good fit of the data in a rather large range of magnetic field from $B_{1}=0.15$ to $B_{2}=0.45 \mathrm{mT}$, as shown in Fig. 2. In this range, we can assume that the magnetic dipole moments carried by the beads have a fixed orientation along $\hat{z}$. We can observe in Fig. 2 that the potential is anharmonic at low magnetic field but harmonic at high field, where the motion of the beads becomes more confined to the vicinity of the minimum of the potential.

Having well characterized the fluctuations of this system at equilibrium, we now investigate the nonequilibrium fluctuations of the same beads when they are driven by a time-dependent magnetic field. The protocol of magnetic field is a periodic function of period $\tau+\tau_{\text {eq }}$, with $\tau$ and $\tau_{\text {eq }}$ defined in Fig. 1. The explicit time dependence of the protocol is $B(t)=B_{2}+\left(B_{1}-B_{2}\right) \sin ^{2}(\pi t / \tau)$ for $0 \leq t \leq \tau$ and $B(t)=B_{2}$ for $\tau \leq t \leq \tau+\tau_{\text {eq }}$. The time $\tau_{\text {eq }}$ represents

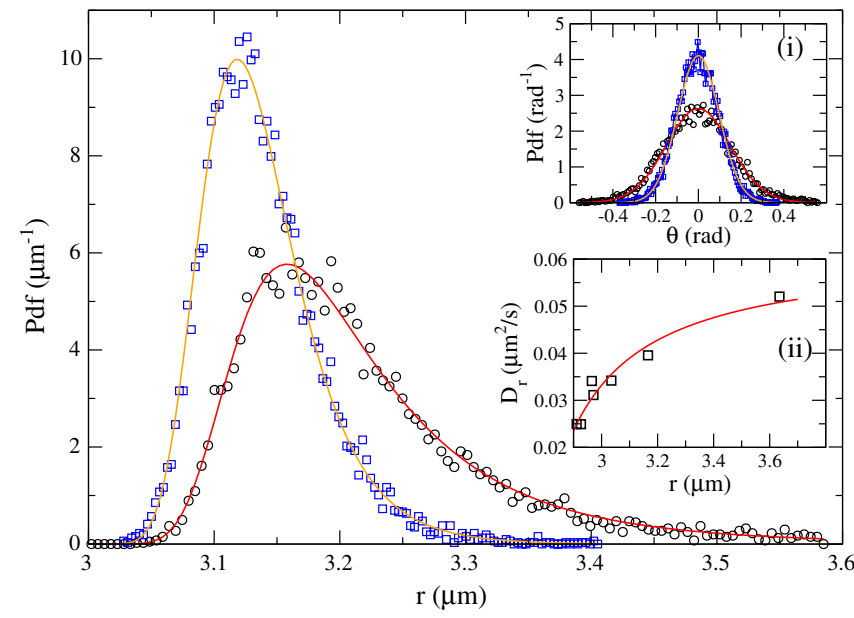

FIG. 2 (color online). Probability distribution function (pdf) of the relative distance between the beads for two values of the magnetic field, namely $B=0.3 \mathrm{mT}$ (circles) and $B=0.45 \mathrm{mT}$ (squares). In inset (i), the pdf of the angle $\theta$ is shown for these two magnetic fields with corresponding symbols, and in (ii), the measurements of the radial diffusion coefficient $D_{r}(r)$ (symbols) are shown as a function of the distance between the beads $r$ (in unit $\mu \mathrm{m}$ ), together with the theoretical prediction using Eq. (3) (solid line). the duration of a pause that is needed to prepare the system at equilibrium for the beginning of the next cycle.

We focus on the dynamics of the displacement vector $\mathbf{r}=(r, \theta)$, which we describe with the following overdamped Langevin equations:

$$
\begin{aligned}
& \Gamma_{r}(r) \dot{r}=f_{r}-\partial_{r} U(r, \theta, B)+\eta_{r} \circ \sqrt{2 k_{B} T \Gamma_{r}(r)}, \\
& \Gamma_{\theta}(r) \dot{\theta}=f_{\theta}-\partial_{\theta} U(r, \theta, B)+\eta_{\theta} \circ \sqrt{2 k_{B} T \Gamma_{\theta}(r)},
\end{aligned}
$$

where $\circ$ denotes the Stratonovich product, $\Gamma_{r}(r)$ and $\Gamma_{\theta}(r)$ denote friction coefficients, and $\eta_{i}$ is a white noise with $i \equiv\{r, \theta\}$ such that $\left\langle\eta_{i}(t) \eta_{j}\left(t^{\prime}\right)\right\rangle=\delta_{i j} \delta\left(t-t^{\prime}\right)$. Drift terms $f_{r}=-\partial_{r} \ln \Gamma_{r}(r) / 2$ and $f_{\theta}=-\partial_{\theta} \ln \Gamma_{\theta}(r) / 2$ are chosen such that the dynamics converge toward equilibrium for a constant magnetic field $[11,12]$.

Dissipation in this system is mainly of hydrodynamic origin. In view of the proximity of the two beads with respect to each other and to the wall, one can rely on the lubrication approximation to describe the hydrodynamic friction coefficients. These coefficients are the sum of the friction due to the sphere-sphere interaction $\Gamma_{i}^{s}$ and the friction between the sphere and the bottom wall $\Gamma_{i}^{w}$. More explicitly $\Gamma_{i}(r)=\Gamma_{i}^{s}+\Gamma_{i}^{w}$ for $i \equiv\{r, \theta\}$, with

$$
\begin{array}{rlrl}
\Gamma_{r}^{s}(r) & =\frac{\gamma a}{4 r-8 a}, & \Gamma_{r}^{w}(r) & =\frac{8}{15} \gamma \ln \frac{a}{b}, \\
\Gamma_{\theta}^{s}(r) & =\frac{\gamma r^{2}}{2 k(r)}, & \Gamma_{\theta}^{w}(r)=\frac{8}{15} \gamma r^{2} \ln \frac{a}{b},
\end{array}
$$

where $a$ is the bead radius, $b$ is the distance between the beads and the wall, $\gamma$ is the bare friction coefficient of a single bead far from the wall, and $k(r)$ is a function given in the Supplemental Material [10] and in Ref. [13].

In order to test this model, we have measured experimentally the radial time autocorrelation function. The short time behavior of this function gives the radial diffusion coefficient $D_{r}(r)=k_{B} T / \Gamma_{r}(r)$. The data points can be well fitted to Eq. (3), as shown in the inset (ii) of Fig. 2. From this fit, one finds that the diffusion coefficient of a single bead far from the wall is $D_{0}=k_{B} T / \gamma=0.12 \mu \mathrm{m}^{2} \mathrm{~s}^{-1}$. This value is rather close to the Stokes-Einstein estimate $0.15 \mu \mathrm{m}^{2} \mathrm{~s}^{-1}$ for a bead of diameter $2.805 \mu \mathrm{m}$ in water.

Within this framework, we study the distributions of thermodynamic quantities like work $W$ and heat $Q$, defined at the trajectory level by [14]

$$
\begin{aligned}
W(\tau) & =\int_{0}^{\tau} d t \dot{B}(t) \partial_{B} U(\mathbf{r}(t), B(t)), \\
Q(\tau) & =\int_{0}^{\tau} d t \nabla_{\mathbf{r}} U(\mathbf{r}(t), B(t)) \circ \dot{\mathbf{r}} .
\end{aligned}
$$

In order to make sure that the system is well equilibrated with a sufficient duration of the pauses, we have compared the equilibrium heat fluctuations $P_{\text {eq }}(Q)$ in a constant 
magnetic field with the internal energy fluctuations $P(\Delta U)$ evaluated in the out of equilibrium experiment, where $\Delta U$ represents the difference of internal energy between the end and the beginning of the cycle. If the system is well equilibrated, both distributions $P_{\text {eq }}(Q)$ and $P(\Delta U)$ should look identical, as they do in Fig. 3 of the Supplemental Material [10].

Using experimental trajectories corresponding to $\tau=2 \mathrm{~s}$, we find an average work of $3.3 \pm 0.2 k_{B} T$ and a standard deviation of $3.6 k_{B} T$. Note that $\langle W\rangle \geq 0$, as expected from the second law of thermodynamics, which imposes that the dissipated work, $W_{\text {diss }}=W-\Delta F$, be on average positive. In the present case, $W_{\text {diss }}=W$ since the free energy difference $\Delta F=0$ for this symmetric protocol. We have also evaluated the distribution of the work $P(W)$ represented in Fig. 3, which is non-Gaussian and agrees with the simulations of Eq. (2). We denote $\beta=1 / k_{B} T$. In the inset, we show that $P(W)$ satisfies the Crooks relation [15]

$$
\ln \frac{P(W)}{P(-W)}=\beta W
$$

both for the experimental data and for the simulations. We observe that the relation holds in a smaller range for the experimental data than for the simulations data due to a lack of statistics in the experiment (460 trajectories in the particular experiment of Fig. 3).

In this figure, we also compare simulations of the work distributions with the state-dependent diffusion coefficient given by Eq. (3) and with a constant diffusion coefficient $D=0.03 \mu \mathrm{m}^{2} \mathrm{~s}^{-1}$, corresponding to a typical distance

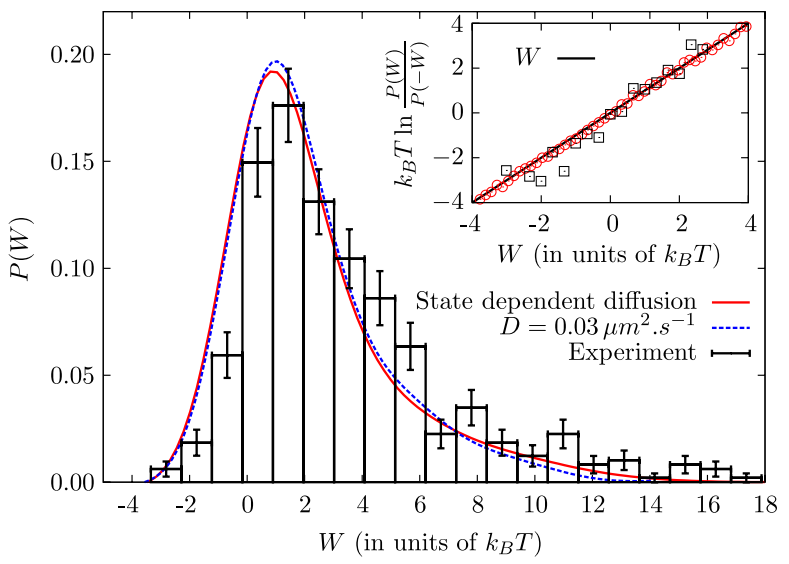

FIG. 3 (color online). Probability distribution of the work $P(W)$, constructed from an experiment using 460 cycles (histogram) and from simulations of Eqs. (2) and (3) with state-dependent diffusion coefficient (red solid line) and with a constant diffusion coefficient $D=0.03 \mu \mathrm{m}^{2} \mathrm{~s}^{-1}$ (blue dashed line). In this experiment, $\tau=2 \mathrm{~s}, \tau_{\text {eq }}=4 \mathrm{~s}$ and the sampling frequency is $40 \mathrm{~Hz}$. The inset shows the verification of the Crooks relation, namely the straight solid line, together with the experimental data (squares) and the simulation results (circles). between the beads. This comparison shows that the distribution of work is only weakly sensitive to the space dependence of the diffusion coefficient. We attribute this to the small excursion experienced by the particles in the cycle. In principle, if this excursion could be made larger while still maintaining a sufficient confinement for a cyclic operation to be possible, one may observe a stronger impact of the space dependence of the friction coefficient on thermodynamic quantities. In any case, the present study of stochastic thermodynamics in the presence of a spacedependent friction represents our first result.

Until now, $\langle W\rangle$ could only be evaluated from the interaction potential, and such a determination had to be very accurate because a relatively small change of the control parameter can produce large variations of this potential. In view of this limitation, it would be interesting to develop alternative methods to estimate the average work, which would ideally bypass the need of a precise calibration of the potential. Since $\langle W\rangle$ is simply related to the average dissipation (the system is in contact with a single thermostat), what we need in fact is an estimate of the average dissipation using only trajectory information. On the theoretical side, it has been established that $\left\langle W_{\text {diss }}\right\rangle$ is related to the determination of an arrow of time, by Jarzynski [16] and by Kawai et al. [17] for Hamiltonian systems and by Crooks [15] and Gaspard [18] for systems in contact with a reservoir. In this latter case and for a reservoir of temperature $T$, one has the following equality:

$$
\beta\left\langle W_{\text {diss }}\right\rangle=D\left(P_{F}\left[c^{F}\right] \| P_{R}\left[c^{R}\right]\right),
$$

where $D\left(P_{F} \| P_{R}\right)$ represents the Kullback-Leibler (KL) divergence between the forward path probability $P_{F}$ evaluated on the forward trajectory $\left[c^{F}\right]$ and the corresponding probability distribution $P_{R}$ evaluated on the backward trajectory $\left[c^{R}\right]$.

In contrast to early studies on fluctuations theorem, which "verified" a relation like Eq. (5) from a determination of $P(W)$ as done in Fig. 3, the above result suggests doing just the opposite: namely enforce the fluctuation relation as a constraint and estimate from it the dissipation using trajectories information. This is indeed possible, as shown in [19], via a careful analysis of the continuous times series of nonequilibrium stationary fluctuations. For discrete time series, a similar idea was put forward in [20] and recently used by two of us for estimating in a noninvasive way the dissipation present in chemical reactions [21]. So far, these ideas have not been exploited experimentally for driven nonstationary systems or for colloids in nonharmonic potentials.

In order to do so, we now project the path probabilities $P_{F}\left[c^{F}\right]$ onto the probability distribution evaluated at a single point at time $t$ in the trajectory, namely $p_{F}(t)$. Similarly, $P_{R}\left[c^{R}\right]$ is projected onto the distribution evaluated at the 
time-symmetric point $p_{R}(\tau-t)$. As a result of these projections, Eq. (6) becomes the following inequality [22], which holds for any times $t$ and $\tau$ :

$$
\beta\left\langle W_{\text {diss }}(\tau)\right\rangle \geq D\left(p_{F}(t) \| p_{R}(\tau-t)\right)
$$

Now, taking advantage of the symmetry of the protocol, we can evaluate $p_{R}$ from the forward protocol. In other words, we record the trajectory at only two points in the cycle: the first one at a time $t$ after the beginning of the cycle and the second one at a time $\tau-t$ as shown in Fig. 1. We use only the information contained in the relative distance between the beads $r$ instead of $(r, \theta)$ since our numerical simulations indicate that reliable estimates of the work can already be obtained in this way. The probability distribution is determined from the experimental data after binning the trajectories, and from these, the KL bound is evaluated as shown in Fig. 4(a). Note that by construction the bound is zero at $t=1 \mathrm{~s}$ where both measurement points merge into a single point. More interestingly, there is a maximum in this bound that occurs roughly halfway through the second half of the protocol at a time $t=1.6 \mathrm{~s}$, and at this point a value of about $1.5 k_{B} T$ is obtained. The precise value of the average dissipation depends on how the KL divergence is evaluated. While all the estimators agree with each other when $t \leq 1 \mathrm{~s}$, a notable difference between them is present near the maximum at $t \simeq 1.6 \mathrm{~s}$, where a log divergence occurs in the data due to lack of statistics.
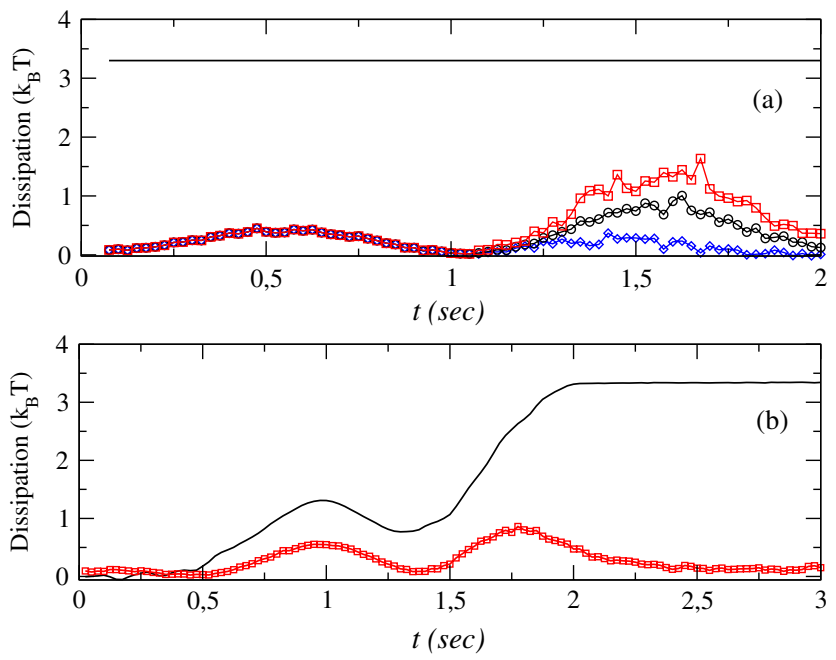

FIG. 4 (color online). (a) Energy-based estimate of dissipation expressed in units of $k_{B} T$ (horizontal solid line) compared with an information-based estimate based on Eq. (7) (symbols) versus time $t$. The three estimators correspond to discarding the points where a log-divergence occurs (blue diamonds), using an adapted binning (black circles), or adding a small bias equal to $1 / 460$ to prevent log divergences (red squares). (b) Energy-based estimate of the dissipated work (black solid line) compared with an information-based estimate based on Eq. (8) (red squares).
The simplest strategy, namely to discard the points where a log-divergence occurs, gives the lowest estimate of the KL divergence. Alternatively, one can either bin the data in such a way that these divergences do not occur or introduce a small constant bias in the probabilities equal to $1 / 460$ in order to remove the divergences. Both methods lead consistently to a higher value, in the range of 1 to $1.5 k_{B} T$ near this maximum. It is also important to appreciate that for all times $t$ considered, the KL bound is always smaller than the value obtained from the "energetic" estimate using the potential, which gives the constant $3.3 \pm 0.2 k_{B} T$, independent of $t$. This is expected since most of the information contained in the trajectories has been discarded in the projection step to obtain Eq. (7), and only the values at two symmetric points were kept. This loss of information represents a form of coarse graining, which is known to lead to an underestimation of the dissipation $[17,22,23]$.

Since an equilibrium probability distribution is typically better known experimentally than its nonequilibrium counterpart, one may be tempted to replace the above comparison between forward and backward nonequilibrium probabilities, by a comparison between a nonequilibrium probability distribution, $p_{\text {neq }}(t)$, with its equilibrium counterpart, $p_{\text {eq }}(t)$. In such a formulation, the equilibrium distribution must be evaluated at the value of the control parameter at time $t$ and compared with the nonequilibrium distribution at the same time $t$ according to [24]:

$$
\beta\left\langle W_{\text {diss }}(t)\right\rangle \geq D\left(p_{\text {neq }}(t) \| p_{\text {eq }}(t)\right),
$$

where $\left\langle W_{\text {diss }}(t)\right\rangle$ is the average dissipative work evaluated up to time $t$. Note that for our specific experimental conditions, Eq. (8) is only a particular case of Eq. (7) when the time $t=\tau$. In Fig. 4(b), both sides of the inequality of Eq. (8) are evaluated for the same experimental data used in Fig. 4(a) as explained in more details in the Supplemental Material [10]. At large time $t,\left\langle W_{\text {diss }}(t)\right\rangle$ tends toward the average work determined before. At short time $t$, both $\left\langle W_{\text {diss }}(t)\right\rangle$ and $D\left(p_{\text {neq }}(t) \| p_{\text {eq }}(t)\right)$ should tend to zero, but a small nonzero value is found in the latter quantity. We attribute this discrepancy to a small error in the determination of the interaction potential that enters in $p_{\text {eq }}$. More importantly, the KL bound reaches a maximum of the order of $1 k_{B} T$ at a time $t \simeq 1.8 \mathrm{~s}$, so somewhere within the second half of the cycle. Therefore, the amplitude of the estimated dissipation and its location in time are both consistent with the determination based on Eq. (7).

To summarize, we have performed a test of stochastic thermodynamics in a system with a space-dependent friction, a friction that we have measured experimentally and characterized with a hydrodynamic model. In a first step, we have followed an energetic approach based on the determination of an interaction potential. In many complex 
systems, this energy-based approach is not practical because the precise determination of the potential is too cumbersome or simply because there are too many variables involved. To address this fundamental issue, we have investigated in a second step, information-theoretic estimations of the average dissipation. Of particular interest is the general formulation based on Eq. (6), which has the advantage of not requiring any knowledge of the energetics of the system or of its equilibrium behavior. Both estimates are lower than the expected level of dissipation, and to improve upon this, extensions of this method are needed to take advantage of the complete information contained in the trajectories as opposed to only the information in a few points as done here. Despite this limitation, informationtheoretic estimates are attractive since they are simple to implement and do not require any knowledge of the dynamics of the system, a definitive advantage for many experimental applications. In particular, we envision that this method could be useful for the monitoring of small chemical or biochemical reactors [21] or for microrheology studies of biological systems.

We acknowledge insightful discussions with H. Stone, J. M. R. Parrondo, and M. Esposito. D. L. also thanks the Kavli Institute for Theoretical Physics China, CAS, Beijing 100190, China, for hospitality where part of this work was done.

[1] U. Seifert, Rep. Prog. Phys. 75, 126001 (2012).

[2] C. Jarzynski, Annu. Rev. Condens. Matter Phys. 2, 329 (2011).

[3] S. Ciliberto, S. Joubaud, and A. Petrosyan, J. Stat. Mech. (2010) P12003.

[4] F. Ritort, Adv. Chem. Phys. 137, 31 (2008).
[5] A. Imparato, L. Peliti, G. Pesce, G. Rusciano, and A. Sasso, Phys. Rev. E 76, 050101 (2007).

[6] V. Blickle, T. Speck, L. Helden, U. Seifert, and C. Bechinger, Phys. Rev. Lett. 96, 070603 (2006).

[7] A. Celani, S. Bo, R. Eichhorn, and E. Aurell, Phys. Rev. Lett. 109, 260603 (2012).

[8] C. Brangbour, O. du Roure, E. Helfer, D. Démoulin, A. Mazurier, M. Fermigier, M.-F. Carlier, J. Bibette, and J. Baudry, PLoS Biol. 9, e1000613 (2011).

[9] D. Lacoste, C. Brangbour, J. Bibette, and J. Baudry, Phys. Rev. E 80, 011401 (2009).

[10] See Supplemental Material at http://link.aps.org/ supplemental/10.1103/PhysRevLett.112.180604 for more information on various aspects of the modelling of this experiment.

[11] A. W. C. Lau and T. C. Lubensky, Phys. Rev. E 76, 011123 (2007).

[12] N. Van Kampen, Stochastic Processes in Physics and Chemistry (North-Holland Personal Library, Amsterdam, 2007).

[13] D. J. Jeffrey and Y. Onishi, J. Fluid Mech. 139, 261 (1984).

[14] K. Sekimoto, Prog. Theor. Phys. 130, 17 (1998).

[15] G. E. Crooks, Phys. Rev. E 61, 2361 (2000).

[16] C. Jarzynski, Phys. Rev. E 73, 046105 (2006).

[17] R. Kawai, J. M. R. Parrondo, and C. Van den Broeck, Phys. Rev. Lett. 98, 080602 (2007).

[18] P. Gaspard, J. Stat. Phys. 117, 599 (2004).

[19] D. Andrieux, P. Gaspard, S. Ciliberto, N. Garnier, S. Joubaud, and A. Petrosyan, J. Stat. Mech. (2008) P01002.

[20] É. Roldán and J. M. R. Parrondo, Phys. Rev. Lett. 105, 150607 (2010).

[21] S. Muy, A. Kundu, and D. Lacoste, J. Chem. Phys. 139, 124109 (2013).

[22] J. Horowitz and C. Jarzynski, Phys. Rev. E 79, 021106 (2009).

[23] M. Esposito, Phys. Rev. E 85, 041125 (2012).

[24] S. Vaikuntanathan and C. Jarzynski, Europhys. Lett. 87, 60005 (2009). 